Cahiers $d u$ MONDE RUSSE

\section{Cahiers du monde russe}

Russie - Empire russe - Union soviétique et États indépendants

$49 / 4 \mid 2008$

Destins individuels et terreur. Jeunesse dans la société post-stalinienne

\title{
Lewis H. Siegelbaum, Cars for Comrades
}

\author{
Larissa Zakharova
}

\section{(2) OpenEdition}

Journals

Édition électronique

URL : https://journals.openedition.org/monderusse/6969

DOI : 10.4000/monderusse.6969

ISSN : $1777-5388$

Éditeur

Éditions de l'EHESS

Édition imprimée

Date de publication : 28 décembre 2008

Pagination : 784-788

ISBN : 978-2-7132-2197-2

ISSN : $1252-6576$

Référence électronique

Larissa Zakharova, "Lewis H. Siegelbaum, Cars for Comrades ", Cahiers du monde russe [En ligne], 49/4 | 2008, mis en ligne le 24 décembre 2009, consulté le 02 septembre 2022. URL : http://

journals.openedition.org/monderusse/6969; DOI : https://doi.org/10.4000/monderusse.6969

Ce document a été généré automatiquement le 2 septembre 2022

Tous droits réservés 


\title{
Lewis H. Siegelbaum, Cars for Comrades
}

\author{
Larissa Zakharova
}

\section{RÉFÉRENCE}

Lewis H. SIEGELBAUM, Cars for Comrades. The life of the Soviet Automobile. Ithaca-

Londres : Cornell University Press, 2008, 309 p.

1 Lewis H. Siegelbaum présente l'histoire d'un moyen de transport, la voiture, dans le $\mathrm{xx}^{\mathrm{e}}$ siècle russe. Bien au-delà d'une histoire industrielle, il s'agit d'une contribution à l'histoire économique, sociale, politique et des relations avec l'étranger, sous l'angle des transferts de technologie, et dans la lignée d'un courant historiographique qui vise à explorer la modernité de l'URSS. L'approche adoptée par l'auteur révèle toutes les contradictions de la civilisation soviétique. Métaphore de la transformation révolutionnaire, objet associé au pouvoir et même à la terreur (les voronki noires du NKVD emmenant leurs victimes dans la nuit), produit élitiste des cinquante premières années du régime, l'automobile incarne les inégalités d'une société conçue pourtant comme égalitaire. Contrairement au transport en commun, la voiture, objet de désir personnel, espace privé mobile, n'est pas un symbole de la marche de l'URSS vers le communisme. Aussi, malgré le progrès technologique et d'importants investissements, les idéaux collectivistes freinent-ils la production automobile de masse, même dans les années 1950-1960 où la consommation individuelle est légitimée. En résulte une révolution tardive dans ce domaine, qui n'aura lieu que dans les années 1970 et aboutit enfin à sa démocratisation. Mais la pénurie chronique de pièces de rechange et d'espaces de stationnement montre combien il est difficile de prévoir jusqu'au bout les conditions nécessaires à un usage généralisé de la voiture.

2 L'ouvrage est bâti sur un corpus solide de divers documents publiés (journaux, magazines spécialisés), de matériaux issus d'archives centrales et régionales, ainsi que sur des sources littéraires et filmographiques. Il est composé de six chapitres. Les trois 
premiers retracent successivement l'histoire de trois usines : AMO-ZIS-ZIL (Moscou), GAZ (Nijni Novgorod) et VAZ (Togliatti). Les transformations postsoviétiques y sont incluses. Le quatrième chapitre traite des routes, les deux suivants brossent le tableau de la répartition des voitures, respectivement pour l'entre-deux-guerres et l'après-guerre. Le choix de cette structure paraît judicieux dans la mesure où le début de l'ouvrage introduit le lecteur dans l'univers spécifique de trois sites de l'industrie automobile, distincts par leurs objectifs et leurs conditions de production, de travail et de vie et correspondant à des époques différentes. En présentant les modèles issus de ces trois usines, l'auteur prépare le lecteur à suivre ces véhicules sur les routes soviétiques, par les villes, plus rarement par les campagnes, et à cerner l'imaginaire des individus qui, rêvant d'en posséder, déploient diverses tactiques pour satisfaire cette envie avant de se trouver confrontés aux problèmes d'entretien de cet objet fétiche.

L'histoire des trois usines est une reconstitution de la civilisation soviétique à la Stephen Kotkin. Le point commun entre les trois sites est l'érection d'une ville autour de l'usine, construite à partir d'un projet particulier de socgorod. La destination élitiste des voitures fabriquées à $\mathrm{GAZ}$ contraste fortement avec les conditions de vie misérables des ouvriers. Les quartiers d'AMO-ZIS sont mieux aménagés, tandis que Togliatti offre un confort incomparable. Dans les trois villes, pourtant, un fossé sépare le cadre de vie et l'approvisionnement en biens de consommation des étrangers et des Soviétiques.

AMO-ZIS-ZIL, entreprise fondée en 1916, connait, après sa nationalisation, une expérience d'importation de Detroit à Moscou sous l'égide d'un groupe de réémigrants russes américains ayant travaillé chez Ford. L'histoire de l'usine suit les soubresauts de l'histoire politique du pays: ses années glorieuses correspondent à l'ère stalinienne, tandis que la déstalinisation la frappe durement. Après le $\mathrm{XX}^{\mathrm{e}}$ Congrès, elle change de nom (l'usine Stalin devient l'usine Lihačev), et frigidaires et vélos remplacent sur ses chaînes les voitures de luxe destinées à transporter les dirigeants. GAZ, à Nijni Novgorod, fondée quant à elle pendant le premier plan quinquennal, concurrence pour un laps de temps AMO-ZIS grâce, là encore, à un partenariat avec Ford. Les premières voitures fabriquées à GAZ en 1945, les Pobeda, ont une forte connotation symbolique, car elles incarnent la victoire de l'URSS. Ce sont en principe les premiers véhicules que de simples citoyens peuvent acquérir. Les Volga (GAZ-21), fabriquées à partir de 1956, deviennent ensuite des objets irremplaçables du paysage urbain des années 1960, au service des fonctionnaires et des taxis. Elles assurent aussi la visibilité internationale de l'industrie automobile soviétique grâce aux exportations et aux rallyes. Mais le chef-d'œuvre de cette usine est incontestablement la Čajka, l'auto la plus grande et la plus « exubérante » de l'après-guerre, apparue dans le contexte de la critique du penchant américain pour les grosses voitures (il faut tout de même rattraper et dépasser les États-Unis!) et offerte au parti à l'occasion du XXI ${ }^{e}$ Congrès. 3000 exemplaires de ce véhicule, produit jusqu'en 1978, assurent la mobilité du corps diplomatique, des fonctionnaires haut placés et de quelques personnalités éminentes, depuis Mihail Šolohov, Jurij Gagarin jusqu'à la ballerine Galina Ulanova. Les modèles réformés continuent leur vie au service de l'Intourist (pour impressionner les touristes étrangers) et des « Palais du mariage » (pour offrir aux nouveaux mariés un peu de rêve socialiste).

Le climat de la guerre froide influe sur le choix des partenaires dans les transferts de technologie occidentale. Si les Volga fabriquées à GAZ dans les années 1970 utilisent des moteurs dérivés de la Peugeot, toute l'histoire de l'usine VAZ à Togliatti, du nom du dirigeant du PCI, incarne la collaboration soviéto-italienne. L’accord signé en 1966 avec 
Fiat, le plus important du point de vue commercial dans l'histoire de l'URSS, doit permettre d'accroître l'offre dans un pays où le nombre de véhicules - 1 voiture pour 238 personnes - est peu flatteur pour l'URSS, surtout en comparaison avec les États-Unis (1 pour 2,7). La mise en place en 1965 d'un ministère de la Production automobile témoigne également d'une prise de conscience de la situation catastrophique dans ce domaine. L'intérêt de Fiat dans cette affaire relève du prestige : l'absence de profit commercial est compensée par le fait de vendre et de construire en URSS. Mais cela ne va pas sans la crainte que les fournitures italiennes soient détournées à des fins militaires par les Soviétiques. Ainsi, bien que la version soviétique de la Fiat-124, la Žiguli, soit bien associée dans l'imaginaire collectif à la production de masse de voitures pour citoyens ordinaires, et non pour l'armée et les dirigeants, les craintes des entrepreneurs italiens sont fondées sur le penchant des autorités soviétiques à tirer un usage particulier du développement de l'industrie automobile.

$\mathrm{Du}$ fait de la priorité accordée à l'industrie lourde par rapport à l'industrie de consommation, la production des camions reste dominante jusqu'en 1972. Le même souci d'industrialiser incite également à privilégier la construction des chemins de fer sur l'aménagement du réseau routier. Cercle vicieux, la faible quantité d'automobiles n'encourageant pas l'aménagement d'artères routières appelées à rester vides de toute façon. Cette conception de l'inefficacité des investissements mène à recourir à des moyens spécifiques pour l'entretien des routes: travail forcé (trudpovinnost') et utilisation d'une main-d'œuvre captive. Les courses automobiles attirent pourtant l'attention sur l'état des routes. C'est seulement à partir de la deuxième moitié des années 1930, à la suite des purges à la Direction de la construction des routes et à l'intégration de cette structure administrative au sein du NKVD, que l'importance du rôle économique et stratégique des routes est enfin reconnue.

Plusieurs axes transversaux tissent la trame du livre, chacun présentant un aspect spécifique du système soviétique, tant dans ses dimensions économiques que politique et sociales. Le premier dévoile le phénomène particulier du bricolage à la soviétique. Celuici vise à adapter les modèles de voiture étrangers aux conditions locales de production (pénurie de pièces détachées, absence de savoir-faire technique) et d'usage (climat, état des routes). Le poids de l'économie étatisée et planifiée se fait également sentir dans la stagnation de la créativité et du design de l'industrie automobile, frappée d'inertie comme bien d'autres branches. Certains modèles restent inchangés pendant trente ans, immobilisme qui reflète la paralysie du régime. Absence de concurrence, impossibilité de prendre en compte la demande sociale et incapacité à dépasser le fordisme sont autant de facteurs responsables.

8 Le deuxième axe examine les liens étroits entre l'économie et le pouvoir, chaque nouveau modèle devant être approuvé par le Kremlin. Une telle confusion entre expertise et autorité politique est révélatrice du paternalisme interventionniste des leaders soviétiques dans la sphère économique. Emblèmes du luxe à la soviétique, certains modèles (par exemple, ZIS-101 ou ZIS-110 fabriqués à l'usine AMO-ZIL) se veulent l'incarnation de l'État, une sorte d'État sur roues, puisqu'ils sont prévus pour transporter la nomenklatura soviétique. En 1928, 78 \% de tous les véhicules du pays appartiennent à l'État. Certains dirigeants apparaissent soit comme de véritables amateurs de voitures (Stalin dispose de cinq automobiles pour son usage personnel afin de pouvoir en changer tous les jours pour "raisons de sécurité »; Brežnev est un passionné de belles cylindrées), soit au contraire comme des militants de la lutte contre 
l'abus, par les fonctionnaires, des véhicules de service et des chauffeurs personnels (Hruščev). Le principe d'attribution des automobiles est fondé sur un mélange de normes institutionnelles et personnelles. Ainsi, sur la liste des destinataires, les institutions sont suivies par des personnalités célèbres (écrivains, académiciens). À cause de ce système, la voiture est perçue comme un symbole de bien-être matériel et de prestige social malgré le souci du pouvoir qu'elle n'apparaisse pas comme telle.

9 En découle le troisième fil conducteur de l'ouvrage - une histoire de la consommation. Un usage spécifique étant assigné à chacun des modèles, ceux-ci reflètent parfaitement la stratification sociale et symbolique. Sous Stalin, l'attribution d'une voiture est une récompense pour un mérite rendu à la société. Dans les années 1930, les individus ordinaires ne peuvent espérer obtenir une automobile que par le biais de la loterie. Quant aux élites, elles usent largement des requêtes écrites adressées à Molotov. Un accès aussi restreint a des implications directes sur le statut social du conducteur: pendant longtemps, conduire une voiture reste une occupation professionnelle, principalement masculine. Les difficultés rencontrées pour réparer et entretenir une voiture exigeant plus de temps libre que ne le laissent les loisirs d'une activité salariée contribuent à réserver l'auto à un usage strictement professionnel. La pratique plus tardive du taxi au noir peut être interprétée comme la conséquence des limites mal définies entre usage personnel et professionnel.

10 L'auteur se demande aussi si les prix exorbitants ne seraient pas une tactique délibérée de l'État pour limiter l'accès des consommateurs à ces biens (en 1973, le prix d'une Žiguli correspond à 3,5 fois le salaire annuel moyen d'un ouvrier). L'acquisition d'une voiture est en général le fruit de huit années d'économies et d'une attente de quatre à dix ans. Vu les inégalités et la pénurie, le discours officiel n'incite pas à acheter, mais justifie et défend les propriétaires de voitures. Sous Hruščev, les contradictions de la politique de production et de distribution des biens de consommation durables ont pour résultat, d'une part, une augmentation des produits électroménagers accessibles à la population mais, d'autre part, une stagnation du nombre de voitures vendues. Or la production automobile augmente pendant cette période: c'est la part des voitures allant à l'exportation et aux institutions qui croît. En même temps, le mythe socialiste du luxe " démocratisé » et accessible à tous apparaît à travers l'attribution de modèles luxueux au service du taxi. Est ainsi résolu en partie, grâce à cette conception collectiviste et «égalitaire» de leur usage, le dilemme de la propriété personnelle des voitures. Dévolues au transport public, celles-ci incarnent désormais allègrement le « confort » de la vie en URSS. Le système de location est censé aller dans le même sens, mais les défauts de l'économie de pénurie dressent là un obstacle insurmontable.

11 On regrettera que certains sujets ne soient pas abordés dans cet ouvrage passionnant. L'auteur mentionne l'Institut de recherches sur l'automobile sans toutefois entrer dans les détails, bien que la présentation des activités de cet établissement ait pu éclairer davantage le rôle et l'état de la recherche et du développement dans l'industrie automobile. Une attention prêtée aux travaux des économistes sur les normes rationnelles de consommation aurait pu aider à clarifier l'attitude contradictoire relative à la propriété privée de voitures. Le lecteur se demande aussi pourquoi d'autres sites, telle l'usine de construction des Zaporožec, située en Ukraine, qui fabriquait des voitures plus accessibles et destinées par Hruščev aux ouvriers, n'ont pas été étudiés. Cela aurait pu compléter le tableau de la répartition des voitures dans la société en fonction de leur valeur symbolique. Enfin, si la présence des ouvriers et spécialistes 
étrangers sur les différents sites fait l'objet d'une analyse, en constituant un volet de l'histoire de la perception de la civilisation soviétique par les Occidentaux, les séjours des travailleurs soviétiques à l'étranger dans le cadre de formations techniques ainsi que leurs conséquences sur les représentations du socialisme et du capitalisme n'occupent que peu de place dans l'ouvrage. Pourtant cette composante « humaine » des échanges et des transferts industriels serait en partie responsable de l'échec du projet de construction du communisme ${ }^{1}$. Néanmoins, notre envie d'en savoir plus témoigne surtout des mérites d'un auteur qui a su éveiller notre curiosité et offrir de stimulantes pistes de réflexion sur le rôle que jouent, dans les interactions sociales, politiques et économiques, certains objets matériels.

\section{NOTES}

1. Nordica Nettleton, "Driving toward communist consumerism. AvtoVAZ », Cahiers du Monde russe, 47 (1-2), janvier-juin 2006, p. 131-149. 